\title{
Técnicas Transobturadoras na Incontinência Urinária de Esforço Feminina
}

\author{
Transobturator Slings for Female Stress Urinary Incontinence
}

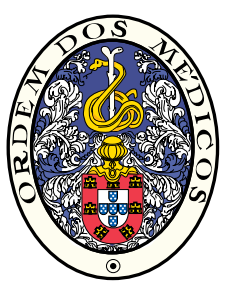

\author{
Ana SOUSA ${ }^{1}$, André JESUS ${ }^{1}$, Maria CARVALHO ${ }^{1}$, Giselda CARVALHO ${ }^{1}$, João MARQUES ${ }^{1}$, Francisco FALCÃO ${ }^{1}$, Isabel \\ TORGAL 1,2 \\ Acta Med Port 2014 Jul-Aug;27(4):422-427
}

\section{RESUMO}

Introdução: A incontinência urinária de esforço afeta 20 a $40 \%$ das mulheres. Os slings colocados sob a uretra média, aplicados por via transobturadora, são a terapêutica consensualmente aceite na atualidade. O objetivo do estudo foi avaliar a eficácia e possíveis complicações associadas à aplicação dos slings colocados por via transobturadora no tratamento da incontinência urinária de esforço. Material e Métodos: Avaliaram-se retrospetivamente 363 doentes submetidas a cirurgia de incontinência urinária de esforço por via transobturadora, nos Hospitais da Universidade de Coimbra do Centro Hospitalar e Universitário de Coimbra, no período de 1 Janeiro de 2008 a 1 Julho de 2010.

Resultados: A média das idades das doentes foi de 56 anos [28-86]. Além da correção da incontinência urinária de esforço, em 13,5\% das mulheres foi associada outra cirurgia vaginal. A maioria $(95,3 \%)$ das mulheres apresentou hipermobilidade da uretra. Ocorreram complicações per-operatórias em $0,8 \%$, complicações pós-operatórias imediatas em $5,2 \%$ e complicações pós-operatórias tardias em $15,7 \%$. A taxa de sucesso global foi de $93,7 \%$. A taxa de sucesso nas doentes com uretra fixa foi $77,8 \%$, verificando-se melhores resultados $(94,5 \%)$ naquelas com hipermobilidade da uretra $(p=0,02)$. A taxa de sucesso foi comparável nas doentes com e sem cirurgias vaginais associadas.

Discussão: As técnicas transobturadoras têm taxas de sucesso elevadas, tendo-se tornado o tratamento de primeira linha para as doentes com IUE, independentemente se tratadas pela técnica outside-in (TOT ${ }^{\circledR}$ ) ou pela inside-out (TVT-O ${ }^{\circledR}$ ). Ambas as técnicas foram concebidas com o intuito de evitar a passagem no espaço retropúbico, reduzindo assim o número de complicações.

Conclusão: As taxas de cura para as abordagens transobturadoras oscilam entre 80 e $95 \%$. A taxa de cura aumenta quando o mecanismo responsável pela incontinência urinária de esforço é a hipermobilidade da uretra, contudo não é alterada quando são realizadas concomitantemente outras cirurgias vaginais.

Palavras-chave: Incontinência Urinária de Esforço; Slings Suburetrais.

\section{ABSTRACT}

Introduction: Stress urinary incontinence affects about $20-40 \%$ of women. Treatment with transobturator mid-urethral slings is consensually accepted nowadays. The goal of this study was to evaluate the success rate and most frequent complications of surgical treatment with transobturator mid-urethral slings in stress urinary incontinence.

Material and Methods: This study evaluated 363 patients who underwent correction of stress urinary incontinence with a transobturator tape in Hospitais da Universidade de Coimbra between January 1st 2008 and July 1st 2010.

Results: The mean age of patients was 56 [28-86]. In 13.5\% of women, the correction of stress urinary incontinence was associated with other vaginal surgery. The majority of these women (95.3\%) had urethral hypermobility. Only $0.8 \%$ of women suffered of perioperative complications, $5.2 \%$ of immediate postoperative complications and $15.7 \%$ of late postoperative complications. The global success rate was $93.7 \%$. The success rate in patients with fixed urethra was lower $(77.8 \%)$ comparing with the results of those with urethral hypermobility, being successful in $94.5 \%(p=0.02)$. The success rate was similar in patients with or without vaginal surgeries.

Discussion: Treatment with transobturator mid-urethral slings has high success rates and it became the first treatment chosen to stress urinary incontinence, even if they were treated with the technique outside-in (TOT $\left.{ }^{\circledR}\right)$ or inside-out (TVT-O ${ }^{\circledR}$ ). Both techniques were conceived to avoid passing through the retropubic space, decreasing the complicate matters number.

Conclusions: The cure rates for the transobturator surgical approach range between 80 and $95 \%$. The cure rate increases when the mechanism responsible for the urinary incontinence is urethral hypermobility, although it is not modified when are performed other vaginal surgeries concomitantly.

Keywords: Urinary Incontinence, Stress; Suburethral Slings.

\section{INTRODUÇÃO}

A incontinência urinária de esforço (IUE) é um problema comum, com uma prevalência que varia entre 20 a $40 \%$ das mulheres, com idades compreendidas entre 15 e 64 anos. ${ }^{1,2}$ Tem um enorme impacto psicossocial, não só nas doentes, como também nas suas famílias, que frequentemente sofrem em silêncio. ${ }^{1,3,4}$

De acordo com a International Continence Society, a IUE define-se como toda a situação de perda involuntária de urina associada a esforços, como correr, tossir ou espirrar. $^{4}$

As alterações comportamentais e fisioterapia dirigida ao fortalecimento do pavimento pélvico são terapêuticas médicas utilizadas com o objectivo de corrigir a IUE. Contudo, além de pouco eficazes, raramente são curativas. Desta forma, os tratamentos cirúrgicos, com slings colocados sob uretra média, tornaram-se o tratamento preferencial. ${ }^{5,6}$

1. Serviço de Ginecologia e Obstetrícia. Hospital de São Francisco Xavier. Lisboa. Portugal.

2. Serviço de Ginecologia e Obstetrícia. Faculdade de Medicina. Centro Hospitalar e Universitário de Coimbra. Coimbra. Portugal.

Recebido: 25 de Setembro de 2013 - Aceite: 01 de Abril de 2014 | Copyright $\odot$ Ordem dos Médicos 2014 
Foram publicados dois estudos ${ }^{7,8}$ que revelaram elevadas taxas de cura utilizando a técnica minimamente invasiva chamada tension-free vaginal tape $\left(\mathrm{TVT}^{\circledR}\right)$. Esta técnica consiste na aplicação, por via retropúbica, de um sling sintético (fita de polipropileno), colocado sob a uretra média. Apesar da sua eficácia, esta técnica implica a realização de cistoscopia de controlo, uma maior curva de aprendizagem e um maior número de complicações motivadas pela passagem dos guias metálicos sem controlo visual, pelo espaço retropúbico. ${ }^{2,5}$ Embora a complicação mais frequente seja a perfuração da bexiga, estão descritas outras, que apesar de raras, pela sua gravidade podem ser fatais. São exemplos disso, o atingimento dos grandes vasos pélvicos e a perfuração intestinal..$^{8-10}$ Deste modo, e com o intuito de reduzir estas complicações, foi criada uma via alternativa que consiste na passagem da prótese pelos buracos obturadores. A técnica original foi descrita em 2001 por Delorme, e consistia na passagem de um sling sintético pelos buracos obturadores (transobturator tape, TOT $^{\circledR}$ ) de fora para dentro (outside-in), fazendo suporte na uretra média. ${ }^{11}$ Em 2003, uma nova técnica cirúrgica foi descrita por De Leval, ${ }^{12}$ em que a passagem do sling pela membrana ou músculo obturador era feita de dentro para fora (inside-out), designada tension-free vaginal obturator tape (TVT-O ${ }^{\circledR}$ ).

Os estudos realizados para comparar as técnicas transobturadoras inside-out (TVT-O ${ }^{\circledR}$ ) e outside-in $\left(\right.$ TOT $^{\circledR}$ ) revelaram taxas de sucesso idênticas, superiores a $80 \%$. $^{2,5}$ Assim, por maior facilidade de aprendizagem, a maioria dos centros adotaram as técnicas de suporte da uretra média por via transobturadora como técnicas de eleição para o tratamento da IUE.

Face ao texto que serve de prelúdio, o objetivo do nosso estudo foi avaliar a eficácia e possíveis complicações associadas à aplicação dos slings colocados por via transobturadora no tratamento da IUE.

\section{MATERIAL E MÉTODOS}

Este trabalho consiste num estudo retrospetivo de 363 doentes submetidas a cirurgia de correção da IUE por via transobturadora, no Serviço de Ginecologia dos Hospitais da Universidade de Coimbra, entre 1 de Janeiro de 2008 e 1 de Julho de 2010 com um follow-up de 9 a 39 meses.

Foi realizada a técnica outside-in em 208 doentes e a técnica inside-out em 155, tendo sido colocada em todas as doentes uma prótese sintética de polipropileno, não absorvível. O tipo de prótese foi selecionado de acordo com a preferência do cirurgião, tendo sido colocadas fitas de TVT-O $^{\circledR}$ (Gynecare-Ethicon) Monarc ${ }^{\circledR}$ (American Medical Systems) e Aris ${ }^{\circledR}$ (Coloplast).

Todas as doentes foram avaliadas com uma história clínica ginecológica e submetidas a exame uroginecológico. A história clínica incluiu a idade, o status hormonal (pré ou pós menopausa), os sintomas de incontinência urinária e cirurgias ginecológicas prévias, nomeadamente cirurgias de correcção da IUE.

O diagnóstico de IUE foi baseado nos sintomas da doente (nomeadamente perda involuntária de urina com os esforços), na restante história clínica e no exame uroginecológico. Este incluía exame uroginecológico (testes de stress, Q-tip e Bonney). Perante dúvidas no diagnóstico e em doentes já submetidas a cirurgias de correção da IUE, foram solicitados estudos urodinâmicos.

O prolapso de órgãos pélvicos, quando presente, foi avaliado simultaneamente e classificado usando o sistema de pelvic organ prolapse quantification (POP-Q).

$A$ incontinência urinária mista foi definida pela coexistência da IUE e incontinência urinária de urgência (IUU). Esta última caracteriza-se por ser acompanhada ou imediatamente precedida pelo súbito e forte desejo de urinar.

O diagnóstico de IUE por hipermobilidade da uretra ou por incompetência esfincteriana foi realizado com base no exame uroginecológico e estudo urodinâmico. Foi considerada IUE por hipermobilidade da uretra sempre que se objetivou perda de urina associada a descida do colo vesical e uretra proximal coincidente com o esforço, com um Q-tip com ângulo da uretra ao esforço $>30^{\circ}$. Ao invés, foi considerada IUE por incompetência esfincteriana quando se verificou perda de urina com a manobra de Valsalva e com teste de tosse, associada a uretra fixa. Nas doentes submetidas a estudo urodinâmico, a incompetência esfincteriana foi designada quando se observou perda de urina com a manobra de Valsalva, para pressões inferiores a $60 \mathrm{~cm} \mathrm{H}_{2} \mathrm{O}$, ou quando a pressão máxima de encerramento da uretra foi inferior a $20 \mathrm{~cm}$ de $\mathrm{H}_{2} \mathrm{O} .{ }^{13-15}$

As técnicas cirúrgicas foram realizadas de acordo com o descrito na literatura. Nas situações em que concomitantemente à colocação de prótese suburetral foi realizada outra cirurgia vaginal, a prótese foi colocada em segundo lugar e numa incisão vaginal distinta.

Todas as doentes foram submetidas a uma dose única de antibioterapia com cefazolina, em dose profilática, cerca de trinta minutos antes da cirurgia. Nas doentes alérgicas à penicilina, a profilaxia antibiótica foi feita com ciprofloxacina. Os procedimentos cirúrgicos foram efetuados sob anestesia geral. Em todas as cirurgias foi realizada a hidrodisseção no trajeto dos guias metálicos e o sling foi colocado sem tensão, nunca recorrendo ao teste da tosse. Após remoção da sonda vesical 24 horas após a cirurgia, foram avaliados, de forma sistemática, os dois primeiros resíduos pós-miccionais recorrendo a cateterização uretral. Foi considerado normal esvaziamento vesical, a existência de dois valores inferiores a 20 \% da micção espontânea.

A primeira consulta, após a cirurgia, realizou-se num período de 4 a 6 semanas. As avaliações subsequentes foram efectuadas no $6^{\circ}$ e $12^{\circ}$ mês, passando posteriormente a ter uma periodicidade anual.

Foram consideradas complicações per-operatórias, todas aquelas que ocorreram durante a cirurgia; complicações pós-operatórias imediatas, as que ocorreram durante o período de internamento e complicações pós-operatórias tardias, as que ocorreram no período pós-alta.

Definiu-se como IUU 'de novo' todas as situações em que surgiram pela primeira vez queixas de IUU após realização de cirurgia de correção da IUE; IUU 'agravada', 
todas aquelas que, apresentando queixas prévias de IUU, viram a sua sintomatologia agravada após a cirurgia.

Considerou-se critério de cura, a ausência de queixas subjetivas de IUE após a cirurgia, e perante um teste de stress negativo. Por outro lado, quando o quadro clínico se manteve inalterado, entendeu-se estar perante o insucesso da técnica.

A análise estatística foi realizada recorrendo ao software Statistical Package for Social Sciences ${ }^{\circledR}$ for Windows, versão 20.0 com a realização do teste Chi-Quadrado. O valor $p$ foi considerado significativo quando foi inferior a 0,05 .

\section{RESULTADOS}

Os dados relativos às características das doentes estão sistematizados na Tabela 1. A média de idades das doentes foi de 56 anos. A maioria das doentes era pré-menopausica e tinha IUM. A técnica mais utilizada foi a outside-in. Apenas $5,0 \%$ das doentes já tinham sido submetidas a cirurgia prévia de IUE, pelas diversas técnicas, nomeadamente 1,4\% (cinco doentes) pela colpossuspensão de Burch, 0,8\% (três doentes) pela técnica de tension-free vaginal tape (TVT ${ }^{\circledR}$ ), $0,6 \%$ (duas doentes) pela técnica outside-in, $0,8 \%$ (três doentes) pela técnica inside-out, 0,6\% (duas doentes) pela técnica Kelly-Marrion,0,2\% (uma doente) pela técnica de Marshall-Marchetti-Krantz e 0,2\% (uma doente) por técnica desconhecida. Foi diagnosticada incompetência esfincteriana em $4,7 \%$ das doentes. A Tabela 2 descreve as doentes que foram submetidas concomitantemente a outras cirurgias vaginais. Observaram-se complicações per-operatórias, em apenas $0,8 \%$ das doentes, complicações pós-operatórias imediatas em 5,2\% das doentes e em 15,7\%

Tabela 1 - Características das doentes $(n=363)$

\begin{tabular}{|c|c|}
\hline Idade média & $56 \pm 11,0[28,86]$ \\
\hline \multicolumn{2}{|l|}{ Status de menopausa } \\
\hline Pré-menopausa & $52,9 \%(192)$ \\
\hline Pós-menopausa & $47,1 \%(171)$ \\
\hline \multicolumn{2}{|l|}{ Tipo de incontinência urinária } \\
\hline IUE* pura & $45,2 \%(164)$ \\
\hline $\operatorname{IUM}^{\star *}$ & $54,8 \%(199)$ \\
\hline \multicolumn{2}{|l|}{ Mecanismo da IUE } \\
\hline Hipermobilidade da uretra & $95,5 \%(346)$ \\
\hline Incompetência esfincteriana & $4,5 \%(17)$ \\
\hline \multicolumn{2}{|l|}{ Prolapso urogenital } \\
\hline Com & $45,5 \%(165)$ \\
\hline Sem & $54,5 \%(198)$ \\
\hline \multicolumn{2}{|l|}{ Técnica utilizada } \\
\hline outside-in $\left(\mathrm{TOT}^{\circledR}\right)$ & $57,3 \%(208)$ \\
\hline inside-out (TVT-O ${ }^{\circledR}$ ) & $42,7 \%(155)$ \\
\hline \multicolumn{2}{|c|}{ Cirurgias vaginais concomitantes } \\
\hline Com & $13,5 \%(49)$ \\
\hline Sem & $86,5 \%(314)$ \\
\hline \multicolumn{2}{|l|}{ Cirurgias prévias de IUE } \\
\hline Com & $5,0 \%(18)$ \\
\hline Sem & $95,0 \%(345)$ \\
\hline
\end{tabular}

das utentes foram identificadas complicações pós-operatórias tardias. Relativamente à técnica cirúrgica utilizada, apenas se verificou diferença estatisticamente significativa nas situações de erosão da prótese, que foram mais prevalentes no grupo submetido à técnica inside-out. Os tipos de complicações aparecem sumariados na tabela 3 .

Globalmente, a taxa de sucesso/ cura encontrada foi $93,7 \%$. Todas as doentes cumpriram o follow-up, não se tendo verificado alterações na taxa de cura durante este período de tempo. Particularizando, as taxas de sucesso da técnica outside-in $(91,8 \%)$ e da inside-out $(96,1 \%)$ foram comparáveis $(p=0,72)$. As taxas de cura encontrada nas doentes com $(95,9 \%)$ e sem $(93,3 \%)$ cirurgias vaginais concomitantes foram idênticas $(p=0,753)$. Já as taxas de sucesso nas doentes com incompetência esfincteriana, foi menor $(77,8 \%)$ comparativamente com as doentes com hipermobilidade da uretra $(94,5 \%), p=0,02$.

\section{DISCUSSÃO}

As técnicas transobturadoras têm taxas de sucesso elevadas, comprovadas em ensaios clínicos validados, tendo-se tornado o tratamento de primeira linha para as doentes com IUE, independentemente se tratadas pela técnica outside-in $\left(\mathrm{TOT}^{\circledR}\right.$ ) ou pela inside-out (TVT-O $\left.{ }^{\circledR}\right) .{ }^{16}$ Alguns cirurgiões têm preferências pessoais. Contudo, estudos comparativos destes slings têm revelado eficácias idênticas. ${ }^{17,18}$ No presente estudo, a técnica mais vezes utilizada foi a outside-in em $57,3 \%$ das doentes.

Ambas as técnicas foram concebidas com o intuito de evitar a passagem no espaço retropúbico, que ocorre com a técnica descrita por Ulmsten, tension-free vaginal tape, reduzindo assim o número de complicações.

Ao passar através do buraco obturador diminui-se o risco de lesão de grandes vasos ou perfuração da bexiga. ${ }^{19}$ Embora o risco de perfuração da bexiga com as técnicas transobturadoras seja pequeno, esta complicação foi descrita por alguns estudos. ${ }^{20}$ No nosso estudo, constatou-se a perfuração da bexiga em apenas um caso, no grupo submetido à técnica inside-out. Tratava-se de uma doente com limitação dos movimentos de abdução da coxa, motivada

Tabela 2 - Cirurgias vaginais concomitantes $(n=49)$

\begin{tabular}{lc}
\hline $\mathrm{HV}^{*}$ & $1,1 \%(4)$ \\
$\mathrm{HV}+\mathrm{CPA}^{\star *}$ & $3.0 \%(11)$ \\
$\mathrm{HV}+\mathrm{CPP}{ }^{\pi}$ & $0,3 \%(1)$ \\
$\mathrm{HV}+\mathrm{CPA}+\mathrm{CPP}$ & $1,7 \%(6)$ \\
$\mathrm{CPA}$ & $3,0 \%(11)$ \\
$\mathrm{CPP}$ & $2,5 \%(9)$ \\
$\mathrm{CPA}+\mathrm{CPP}$ & $1,7 \%(6)$ \\
Colpocleisis & $0,3 \%(1)$ \\
\hline $\mathrm{HV}^{*}$ Histerectomia vaginal; $\mathrm{CPA}^{* *}$ Colpoplastia anterior; CPP": Colpoplastia posterior
\end{tabular}


Tabela 3 - Complicações

\begin{tabular}{|c|c|c|c|c|}
\hline & $\begin{array}{l}\text { Globais } \\
(n=363)\end{array}$ & $\begin{array}{l}\text { Técnica Outside-in } \\
\qquad(n=208)\end{array}$ & $\begin{array}{l}\text { Técnica Inside-out } \\
\quad(n=155)\end{array}$ & $p$ \\
\hline Complicações per-operatórias & $0,8 \%(3)$ & $1,0 \%(2)$ & $0,6 \%(1)$ & 0,609 \\
\hline Perfuração vesical & $0,3 \%(1)$ & $0,0 \%(0)$ & $0,6 \%(1)$ & 0,573 \\
\hline Perfuração uretral & $0,3 \%(1)$ & $0,5 \%(1)$ & $0,0 \%(0)$ & 0,427 \\
\hline Hemorragia & $0,3 \%(1)$ & $0,5 \%(1)$ & $0,0 \%(0)$ & 0,573 \\
\hline Complicações Pós-operatórias imediatas & $5,2 \%(19)$ & $4,8 \%(10)$ & $5,8 \%(9)$ & 0,423 \\
\hline Resíduos miccionais elevados & $3,6 \%(13)$ & $3,8 \%(8)$ & $3,2 \%(5)$ & 0,494 \\
\hline Infecção do trato urinário & $1,9 \%(7)$ & $1,9 \%(4)$ & $1,9 \%(3)$ & 0,639 \\
\hline Hematoma & $0,6 \%(2)$ & $0,5 \%(1)$ & $0,6 \%(1)$ & 0,672 \\
\hline Deiscência da sutura & $0,3 \%(1)$ & $0,5 \%(1)$ & $0,0 \%(0)$ & 0,573 \\
\hline Complicações pós-operatórias tardias & $15,7 \%(57)$ & $15,9 \%(33)$ & $15,5 \%(24)$ & 0,521 \\
\hline IUE* & $6,9 \%(25)$ & $8,2 \%(17)$ & $3,9 \%(6)$ & 0,072 \\
\hline IUU** "de novo" & $4,4 \%(16)$ & $4,3 \%(9)$ & $4,5 \%(7)$ & 0,563 \\
\hline IUU agravada & $3,0 \%(11)$ & $2,9 \%(6)$ & $3,2 \%(5)$ & 0,542 \\
\hline Erosão da prótese & $2,8 \%(10)$ & $1,0 \%(2)$ & $5,2 \%(8)$ & 0,018 \\
\hline Resíduos miccionais elevados & $0,8 \%(3)$ & $0,5 \%(1)$ & $1,3 \%(2)$ & 0,301 \\
\hline
\end{tabular}

por uma cirurgia ortopédica prévia. Uma outra complicação per-operatória verificada foi a lesão da uretra, que resultou de uma incisão profunda durante a dissecção da mucosa vaginal. A solução de continuidade foi detectada intra-operatoriamente. A doente manteve-se algaliada durante 10 dias e teve uma boa evolução a posteriori, sem sequelas.

$\mathrm{Na}$ literatura estão ainda descritas outras complicações no pós-operatório imediato, como sejam lesões vasculares, lesões intestinais, hemorragia no trajeto dos slings, retenção urinária e persistência de incontinência. A dificuldade de micção após técnicas transobturadoras varia entre 3 a $13 \% .^{21}$ No nosso estudo a complicação pós-operatória mais frequente foi a presença de resíduos miccionais elevados, que ocorreram em 3,6\% das doentes. Quando estes se mantiveram elevados durante seis semanas após a cirurgia, instituiu-se profilaxia com nitrofurantoína. Não houve necessidade de seccionar o sling em nenhuma delas, com resolução espontânea destes casos. A infeção do trato urinário foi a segunda complicação mais frequente. Este tipo de complicação não é incomum nas cirurgias de IUE. A frequência aumenta com a duração da algaliação, a uma taxa de 6 a 7,5\% por dia. ${ }^{22} \mathrm{Na}$ nossa amostra foram diagnosticadas 1,9\% (sete doentes) de infeções urinárias e todas foram tratadas com antibiótico. Registaram-se dois hematomas no pós-operatório imediato, um em cada grupo. Os hematomas ocorreram no trajecto dos guias metálicos e mediam menos de $3 \mathrm{~cm}$ de maior eixo. Uma destas doentes era portadora de um stent coronário, tendo sido submetida a cirurgia de correção de incontinência urinária sob terapêutica com acido acetilsalicílico. Ambas foram tratadas com anti-inflamatórios e a resolução verificou-se ao fim de dois meses. Durante o internamento foi detetada também uma deiscência da sutura. Em todas as cirurgias de correção da IUE, a mucosa vaginal foi encerrada com
Vicryl rapid $2.0^{\circledR}$, com sutura cruzada e contínua. Tratando-se de uma sutura contínua, com exposição da prótese e no pós-operatório imediato, a doente foi ressuturada sob anestesia local, em ambulatório.

As complicações tardias descritas na literatura são a IUU 'de novo', distúrbios de micção, erosão da fita e recorrência de IUE. Todas estas complicações foram observadas no nosso estudo. A IUU 'de novo' foi observada em $4,4 \%$ das nossas doentes, estando esta percentagem de acordo com a literatura $(1,5$ a $6,7 \%) .{ }^{23}$ Relativamente à erosão da fita, as taxas descritas, variam entre 0,3 a $23 \%{ }^{24}$ No nosso estudo detectaram-se $2,8 \%$ de erosões da mucosa, tendo-se verificado uma maior taxa no grupo submetido à técnica inside-out (oito doentes) e apenas dois casos no grupo outside-in.

A dissecção da mucosa vaginal, efectuada de cada lado da uretra, para a passagem dos guias metálicos, é idêntica em ambas as técnicas. Não deverá ser demasiadamente superficial, pelo risco de erosão, principalmente em mulheres com atrofia vaginal, típica da menopausa. De facto, a maioria das erosões que se constataram, foram fora da linha média, o que leva a supor que a dissecção da mucosa, durante o procedimento técnico, terá sido excessivamente superficial.

Existem vários tratamentos perante situações de erosão, que variam desde procedimentos mais simples, como a sutura da mucosa vaginal, cobrindo a zona de erosão, ${ }^{25}$ até à remoção total ou parcial do sling, com ou sem nova cirurgia de correção da IUE. ${ }^{26,27}$ As nossas doentes com erosão foram submetidas à remoção parcial do sling exposto e sutura da mucosa vaginal, e posteriormente, a tratamento médico com estrogénios tópicos. Oito das doentes foram tratadas sob sedação, uma sob anestesia local, e outra sob anestesia geral em ambulatório. 
As taxas de eficácia descritas na literatura por De Leval ${ }^{12,28}$ e mais tarde por Lee et $\mathrm{al}^{21}$ e Lipis et $\mathrm{al}^{29}$ foram $91 \%, 86,8 \%$ e $87 \%$, respetivamente. Assim, os resultados obtidos no nosso estudo $(93,7 \%)$ foram ligeiramente superiores ao que está descrito na literatura. Verificou-se, no entanto, uma diminuição da eficácia $(77,8 \%)$ nas doentes com IUE por incompetência esfincteriana, relativamente às doentes com hipermobilidade da uretra $(p=0,02)$. De facto, as mulheres com IUE com incompetência esfincteriana e uretra fixa têm uma IUE mais grave e uma menor taxa de sucesso cirúrgico do que as mulheres com IUE e uretra móvel. ${ }^{13,14} \mathrm{~A}$ taxa de sucesso nas doentes com incompetência esfincteriana, é de apenas $70 \%,{ }^{13,30}$ semelhante à amostra em estudo descrita. Há autores que defendem que, para este tipo de IUE, a técnica retropúbica é a opção cirúrgica mais adequada. ${ }^{13,30}$ A explicação assenta nos diferentes eixos dos slings. $\mathrm{O}$ eixo do sling retropúbico é perpendicular ao eixo uretral, contrariamente ao eixo agudo do sling transobturador. Deste modo o sling retropúbico tem a forma de um segmento suspensivo em ' $U$ ' e um estreito ângulo sob a uretra em repouso, criando uma maior compressão circunferencial da uretra e um aumento da pressão uretral. ${ }^{13,14}$

No nosso estudo verificou-se que a taxa de sucesso foi superior no grupo das doentes que realizaram outras cirurgias vaginais concomitantemente. No entanto, esta diferença não foi significativa $(p=0,753)$. Esta constatação é

\section{REFERÊNCIAS}

1. Long RM, Giri SK, Flood HD. Current concepts in female stress urinary incontinence. Surgeon. 2008;6:366-72.

2. Cordeiro A, Lermann R, Grilo I, Martins A. Fitas suburetrais transobturadoras na incontinência urinária de esforço feminina, resultados a médio prazo. Acta Med Port. 2010;23:589-96.

3. Hunskaar S, Lose G, Sykes D, Voss S. The prevalence of urinary incontinence in women in four European countries. BJU Int. 2004;93:324-30.

4. Abrams P, Cardoxo L, Fall M, Griffiths D, Rosier P, Ulmsten U, et al. The standardisation of terminology in lower urinary tract function: report from standardization sub-committee of the International Continence Society. Urology. 2003;61:37-49.

5. Lee KS, Choo MS, Lee YS, Han JYY, Kim JY, Jung BJ et al. Prospective comparison of "inside-out" and "ouside-in" transobturator-tape procedures for the treatment of female stress urinary incontinence. Int Urogynecol J. 2008;19:577-82.

6. Feng $\mathrm{CL}$, Chin $\mathrm{HY}$, Wang $\mathrm{KH}$. Transobturator vaginal tape inside out procedure for stress urinary incontinence: results of 102 patients. Int Urogynecol J. 2008;19:1423-7.

7. Nilsson CG, Kuuva N, Falconer C, Rezapour M, Ulmsten U. Long-term results of the tension-free vaginal tape (TVT) procedure for surgical treatment of female stress urinary incontinence. Int Urogynecol J Pelvic Floor Dysfunct. 2001;12:5-8.

8. Peyrat L, Boutin JM, Bruyere F, Haillot O, Fakfak H, Lanson Y. Intestinal perforation is a complication of TVT procedure for urinary incontinence. Eur Urol. 2001;39:603-5.

9. Fourie T, Cohen PL. Delayed bowel erosion by tension-free vaginal tape (TVT). Int Urogynecol J Pelvic Floor Dysfunct. 2003;14:362-4.

10. Nilsson CG, Falconer C, Rezapour M. Seven-year follow-up of the tension-free vaginal tape procedure for the treatment of urinary continence. Obstet Gynecol. 2004;104:1259-62.

11. Delorm E. Trans-obturator urethral suspension: a minimally invasive procedure to treat female stress urinary incontinence. Prog Urol. 2001;11:1306-13

12. De Leval J. Novel surgical technique for the treatment of female stress urinary incontinence: transobturator vaginal tape inside-out. Eur Urol. 2003;44:724-30.

13. Güngördük K, Celebi I, Ark C, Celikkol O, Yildirim $G$. Which type of mid- também apoiada pela literatura. ${ }^{31}$

Uma das limitações deste trabalho está relacionada com o facto de se tratar de um estudo retrospectivo. Consequentemente, não foi possível obter informação sobre algumas características das doentes, tais como: índice de massa corporal, a doenças concomitantes (diabetes, doenças pulmonares, doenças neurológicas), a hábitos medicamentosos, situações que podem influenciam quer o número e tipo de complicações, quer a taxa de cura destas técnicas.

\section{CONCLUSÃO}

Pelo exposto, podemos concluir que a taxa de complicações das técnicas transobturadoras é inferior às descritas para as técnicas retropúbicas. As técnicas inside-out e outside-in têm resultados semelhantes exceto a erosão que será mais associada à primeira. A taxa de cura diminui quando o mecanismo responsável pela IUE é a incompetência esfincteriana, contudo não é alterada quando são realizadas concomitantemente outras cirurgias vaginais.

\section{CONFLITOS DE INTERESSE}

Não existem quaisquer conflitos de interesse.

\section{FONTES DE FINANCIAMENTO}

Não foram utilizados subsídios ou bolsas para a realização deste trabalho.

urethral sling procedure should be chosen for treatment of stress urinary incontinence with intrinsic sphincter deficiency? Tension-free vaginal tape or transobturator tape. Acta Obstetr Gynecol. 2009;88:920-6.

14. Schierlitz L, Dwyer PL, Rosamilia A, Murray C, Thomas E, De Souza $A$, et al. Effectiveness of tension-free vaginal tape compared with transobturator tape in women with stress urinary incontinence and intrinsic sphincter deficiency. Obstet Gynecol. 2008;112:1253-61.

15. Abrams P, Cardozo L, Fall M, Griffiths D, Rosier P, Ulmsten U, et al. The standardization of terminology of lower urinary tract function: report from the standardization Sub-committee of International Continence Society. Neourol Urodyn. 2002;21:167-8.

16. Al-Singary W, Shergill IS, Allen SE, John JA, Arya M, Patel HR. Trans-obturator tape for incontinence: a 3-year follow-up. Urol Int. 2007;78:198-201.

17. deTayrac R, Deffieux X, Droupy S, Chauveaud-Lambling A, CalvaneseBenamour L, Fernandez $\mathrm{H}$. A prospective randomized trial comparing tension-free vaginal tape and transobturator suburethral tape for surgical treatment of stress urinary incontinence. Am J Obstet Gynecol. 2004;190:602-8.

18. Mellier G, Benayed B, Bretones S, Pasquier JC. Suburethral tape via the obturator route: is TOT a simplification of the TVT? Int Urogynecol J Pelvic Floor Dysfunct. 2004;15:227-32.

19. Daneshgari F, Kong W, Swartz M. Complications of mid urethral sling: important outcomes for future clinical trials. J Urol. 2008;180:1890-7.

20. Minaglia S, Ozel B, Klutke J. Bladder injury during transobturator sling. Urology. 2004;64:376-7.

21. Lee Ks, Han DH, Choi YS, Yum SH, Song SH, Doo CK, et al. A prospective trial comparing tension-free vaginal tape inside-out for surgical treatment of female stress urinary incontinence: 1-year follow-up. J Urol. 2007; 177:214-8.

22. Foucher JE, Marshall V. Nosocomial catheter associated urinary tract infections. Infect Surg. 1983;2:43.

23. Zugor V, Labanaris AP, Rezaei-Jafari MR, Hammerer P, Dembowski J, Witt J, et al. TVT vs. TOT: a comparison in terms of continence results, complications and quality of life after a median follow-up of 48 months. Int Urol Nephrol. 2010;42:915-20.

24. Clemens JQ, DeLancey JO, Faerber GJ, Westney OL, Mcguire EJ. 
Urinary tract erosions after synthetic pubovaginal slings: diagnosis and management strategy. Urology. 2000;56:589-94.

25. Giri SK, Sil D, Narasimhulu G, Flood HD, Skehan M, Drumm J. Management of vaginal extrusion after tension-free vaginal tape procedure for urodynamic stress incontinence. Urology. 2007;69:1077-80.

26. Volkmer BG, Nesslauer T, Rinnab L, Schradin T, Hautmann RE, Gottfried HW. Surgical intervention for complications of tension-free vaginal tape procedure. J Urol. 2003;169:570-4.

27. Mustafa M, Wadic BS. Bladder erosion of tension-free vaginal tape present as vesical stone; management and review of literature. Int Urol Nephrol. 2007;39:453-5.

28. Abdel-fattah M, Ramsay I, Pringle S, Hardwick C, Ali Hassan. Evaluation of transobturator tapes (E-TOT) study: randomised prospective single- blinded study comparing inside-out vs. ouside-in transobturator tapes in management of urodynamic stress incontinence: short term outcomes. Eur J Obstet Gynecol Reprod Biol. 2010;149:106-11.

29. Liapis A, Bakas P, Creatsas G. Monarc vs TVT-O for the treatment of primary stress incontinence: a randomised study. Int Urogynecol J. 2008;19:185-90.

30. Schierlitz L, Dwyer PL, Rosamilia A, Murray C, Thomas E, De Souza A, et al. Effectiveness of tension-free vaginal tape compared with transobturator tape in women with stress urinary incontinence and intrinsic sphincter deficiency. Obstet Gynecol. 2008;112:1253-6.1

31. Ayhan A, Dogan U, Guven S, Guler OT, Boynukalin FK, Salman MC Clinical outcome of transobturator tape concomitant with vaginal hysterectomy plus anterior posterior colporrahaphy. Arch Gynecol Obstet. 2009;280:375-80. 


\section{Técnicas Transobturadoras na Incontinência Urinária de Esforco Feminina}

Publicado pela Acta Médica Portuguesa, a Revista Científica da Ordem dos Médicos

Av. Almirante Gago Coutinho, 151

1749-084 Lisboa, Portugal.

Tel: +351218428215

E-mail: submissao@actamedicaportuguesa.com

www.actamedicaportuguesa.com

ISSN:0870-399X | e-ISSN: 1646-0758

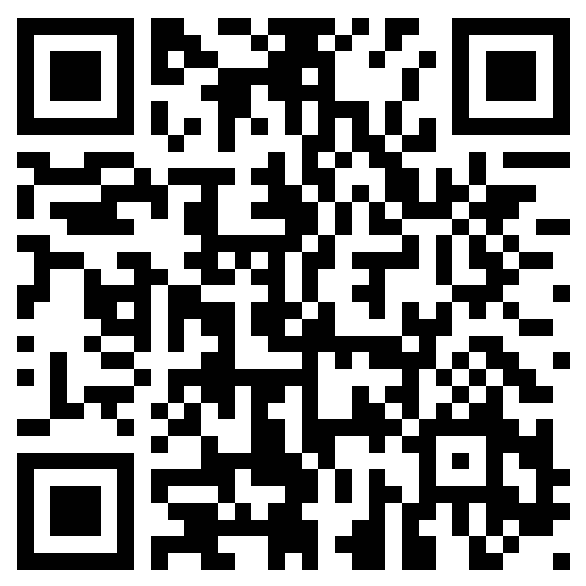

